\title{
Classical demonstration of frequency-dependent noise ellipse rotation using optomechanically induced transparency
}

\author{
Jiayi Qin, ${ }^{*}$ Chunnong Zhao, ${ }^{\dagger}$ Yiqiu Ma ${ }^{\ddagger}$ Xu Chen, Li Ju, and David G. Blair \\ School of Physics, University of Western Australia, Crawley, Western Australia 6009, Australia
}

(Received 20 October 2013; published 22 April 2014)

\begin{abstract}
Cavities with an extremely narrow linewidth of $10-100 \mathrm{~Hz}$ are required for realizing frequency-dependent squeezing to enable gravitational wave detectors to surpass the free mass standard quantum limit over a broad frequency range. High-finesse cavities on the scale of tens of meters have been proposed for this purpose. Optomechanically induced transparency (OMIT) enables the creation of optomechanical cavities in which the linewidth limit is set by the extremely narrow linewidth of a high- $Q$-factor mechanical resonator. Using an 85-mm OMIT cavity with a silicon nitride membrane, we demonstrate a tunable linewidth from $3 \mathrm{~Hz}$ up to several hundred hertz and frequency-dependent noise ellipse rotation using classical light with squeezed added noise to simulate quantum squeezed light. The frequency-dependent noise ellipse angle is rotated in close agreement with predictions.
\end{abstract}

DOI: 10.1103/PhysRevA.89.041802

PACS number(s): 42.50.Wk, 42.25.Hz

Introduction. The coherent interaction of laser radiation with widely spaced mirror test masses is used to measure gravitational-wave-induced motion in interferometric gravitational-wave detectors. The sensitivity of firstgeneration gravitational-wave $(\mathrm{GW})$ detectors such as Laser Interferometer Gravitational-Wave Observatory (LIGO) reached the quantum-shot-noise limit in the high-frequency part of the spectrum. In the second-generation detectors now under construction, quantum radiation-pressure noise is expected to dominate at low frequencies, while shot noise will dominate at high frequencies. A region around $100 \mathrm{~Hz}$ is limited by classical test mass thermal noise, but as better optical coatings and test masses become available, future detectors should be limited mostly by quantum noise.

In the late 1960s, Braginsky pointed out that there exists a standard quantum limit (SQL) in gravitational-wave detectors [1] and proposed that quantum nondemolition (QND) devices could beat the SQL [2]. In 2001, Kimble et al. [3] proposed QND interferometer designs that involved the use of pairs of successive filter cavities for realizing frequencydependent squeezing (FDS) of the input squeezed light or frequency-dependent (FD) homodyne detection in which the output field of the detector is filtered in the frequencydependent way. They pointed out that the sensitivity of such designs across the entire frequency band can be improved below the SQL. Recently Chelkowski et al. demonstrated a FD squeezed vacuum using a short filter cavity in the $\mathrm{MHz}$ range [4]. In 2012, Stefszky et al. demonstrated 11.6-dB squeezing in an Advanced LIGO (aLIGO) detection band [5].

To match the filter cavity linewidth to the corner frequency of ground-based laser interferometers where the shot noise becomes higher than the radiation-pressure noise, the filter cavity must meet very demanding specifications that require very long optical cavities with very low optical losses. To optimize the sensitivity, an adjustable cavity linewidth and

\footnotetext{
*jiayiqinphysics@gmail.com

†chunnong.zhao@uwa.edu.au

${ }^{\ddagger}$ myqphy@gmail.com
}

offset frequency locking are also required. For example, an aLIGO-type GW detector requires filter cavities on the scale of tens of meters with a linewidth of $\sim 100 \mathrm{~Hz}$ to optimize sensitivity [6].

In order to realize FDS in tabletop filter cavities, Ma et al. proposed using the optomechanically induced transparency (OMIT) effect to achieve a tunable narrow linewidth [7]. The idea of OMIT is analogous to the electromagnetically induced transparency (EIT) phenomena discovered in threelevel atomic systems [8]. This phenomenon was widely recognized and applied in various fields of optics [9-12]. Recently, Mikhailov et al. proposed the use of EIT to create a FD squeezed vacuum for GW detectors. However, the high optical loss of EIT is still an issue [13].

The OMIT phenomenon has been studied and demonstrated by various research groups. Weis et al. [14] presented OMIT phenomena in a toroidal microcavity and achieved a tunable linewidth of 50-500 kHz compared with the $15 \mathrm{MHz}$ linewidth of the optical mode. Recently, Karuza et al. [15] demonstrated the OMIT effect in a membrane-in-the-middle setup at room temperature. They reported a maximum signal time advance $\tau^{T} \approx-108 \mathrm{~ms}$ of a probe pulse, which implies an OMIT linewidth much narrower than those mentioned above [14].

In Ref. [7], Ma et al. investigated theoretically the use of optomechanical interactions to achieve tunable narrow linewidth in a tabletop filter cavity. This configuration allows possible realization of FDS in a tabletop experiment.

The experimental challenges of such a device are the stringent demands for very low temperature and a very low-loss optical cavity if optical dilution were used. The optical dilution is realized by using optomechanical interactions to increase the effective $Q$ factor of a given mechanical resonator. To evade quantum backaction of strong optical dilution, Chang et al. [16] and $\mathrm{Ni}$ et al. [17] proposed using a nonlinear quadratic optical trap. Recently, Korth et al. [18] proposed detecting quantum backaction in the outgoing field and actively feeding back to the system. The requirement for a low-temperature operation of such a device is [7]

$$
\frac{8 k_{B} T}{Q_{m}}<\hbar \Gamma_{\mathrm{opt}}
$$


where $T$ is the environmental temperature, $Q_{m}$ is the mechanical $Q$ factor, and $\Gamma_{\text {opt }}$ is the effective cavity linewidth. For an OMIT cavity with $\Gamma_{\mathrm{opt}} \approx 2 \pi \times 100 \mathrm{~Hz}$, the temperature requirement is $T / Q_{m}<6.0 \times 10^{-10} \mathrm{~K}$. In 2008, Zwickl et al. reported that a silicon nitride membrane has mechanical $Q>10^{6}$ at $293 \mathrm{~K}$ and $Q>10^{7}$ at $300 \mathrm{mK}$ [19]. Recently, Jayich et al. observed a mechanical quality factor $Q>4 \times 10^{6}$ of a silicon nitride membrane placed at the center of an optical cavity at $400 \mathrm{mK}[20]$.

In this paper, we use a noise-added signal light to mimic the squeezed light in a room-temperature system in which a control light is injected into the same port for generating the OMIT effect. We demonstrate frequency-dependent noise ellipse rotation in a tunable OMIT cavity in which the linewidth can be tuned from $3 \mathrm{~Hz}$ to several hundred hertz. In [7], a quantum squeezed vacuum is injected into and detected from the reflection port of an OMIT cavity with a highly reflected end mirror. In order to measure the noise ellipse rotation of the single-mode signal field by the lock-in technique [21], we detect the beating of the signal field and the control field at the transmission port of the coupled system. The result proves that the OMIT cavity has the same amplitude and phase response as a simple filter cavity, which can rotate the noise ellipse of a classical signal light with squeezed added noise in close agreement with the theoretical phase response. This shows the potential of FD squeezed vacuum generation in a small-scale compact system with future implementations of low-temperature environment and proper optical dilution.

Optomechanical dynamics. Optomechanical interaction in our setup happens when beating between the control and signal optical fields creates a radiation pressure that induces mechanical motion. This mechanical motion then modulates the control field to produce an upper sideband that has the same frequency as the signal field, but $180^{\circ}$ out of phase, as indicated in Fig. 1. That is, the optomechanical interaction causes the signal field to be reduced and creates the OMIT effect. The linewidth of an OMIT cavity is determined by the sum of the mechanical damping $\gamma_{m}$ and the optomechanical damping $\Gamma_{\mathrm{opt}}$ associated with optomechanical damping of the membrane by a red-detuned control field. Using a mechanical oscillator with a sufficient high- $Q$-factor, this tunable optomechanical damping dominates over mechanical damping and thus sets the linewidth of the system.

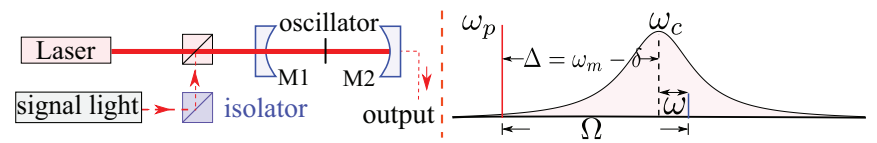

FIG. 1. (Color online) Configuration schematics (left) and frequency relationships. The signal light with squeezed added noise having frequency $\omega$ with respect to the cavity resonance $\omega_{c}$ is injected into an optical cavity with a high- $Q$-factor membrane in the middle that acts as an oscillator at the resonant frequency $\omega_{m}$. The position of the membrane is chosen to introduce a linear optomechanical coupling. The radiation pressure from the beating between the signal light $\omega_{s}=\omega_{p}+\Omega$ and the control laser $\omega_{p}$ coherently drives the mechanical oscillator, which in turns creates sidebands destructively interfering with the signal light, which in effect rotates the noise ellipse angle.
As shown on the right-hand side of Fig. 1, the control laser at frequency $\omega_{p}$ maintains a strong control field $\bar{a}_{p} e^{-i \omega_{p} t}$ in the cavity at the resonant frequency $\omega_{c}$. A weak signal light is injected into the same port (M1) as a small input term $\delta \hat{a}_{\text {in }}=\hat{a}_{\text {in }}^{s} e^{-i \omega_{s} t}$. The frequency difference $\Omega$ between the control field $\omega_{p}$ and signal field $\omega_{s}=\omega_{c}+\omega$ needs to be close to the mechanical resonant frequency $\omega_{m}$ for efficient driving of the mechanical mode.

The Hamiltonian that describes the system here is given by

$$
\hat{H}=\hbar\left(\omega_{c}+G_{0} \hat{x}\right) \hat{a}^{\dagger} \hat{a}+\hat{H}_{m}+\hat{H}_{\gamma} .
$$

Here $\hat{H}_{m}=\hat{p}^{2} / 2 m+m \omega_{m}^{2} \hat{x}^{2} / 2$ is the Hamiltonian of the mechanical oscillator, $H_{\gamma}=-i \hbar \sqrt{2 \gamma_{1}} \hat{a} \hat{a}_{\text {in }}^{\dagger}-i \hbar \sqrt{2 \gamma_{2}} \hat{a} \hat{b}_{\text {in }}^{\dagger}+$ H.c. describes the interaction between the intracavity field $\hat{a}$ and external electromagnetic fields $\hat{a}_{\text {in }}$ and $\hat{b}_{\text {in }}$ with interaction strengths $\gamma_{1}=c T_{1} / 4 L$ and $\gamma_{2}=c T_{2} / 4 L$ through the cavity mirrors $\mathrm{M} 1$ and $\mathrm{M} 2$, respectively, and $G_{0}$ is the linear optomechanical coupling strength [22,23].

Since the signal light $\hat{a}_{\text {in }}^{s}$ in our experiment is a classical field, we neglect the vacuum fluctuation. In the rotating frame at frequency $\omega_{p}$, we have

$$
\begin{aligned}
\chi(\Omega) \hat{x}(\Omega) & =-\hbar \bar{G}_{0}\left[\hat{a}(\Omega)+\hat{a}^{\dagger}(-\Omega)\right]+\hat{F}_{\mathrm{th}}, \\
\hat{a}(\Omega) & =\frac{\bar{G}_{0} \hat{x}(\Omega)}{\Omega-\Delta+i \gamma}+\frac{i \sqrt{2 \gamma_{1}} \hat{a}_{\mathrm{in}}^{s}(\Omega)}{\Omega-\Delta+i \gamma},
\end{aligned}
$$

where $\gamma_{m}$ and $\gamma=\gamma_{1}+\gamma_{2}$ are the linewidths of the mechanical oscillator and the cavity, $\chi(\Omega)=m\left(\omega_{m}^{2}-\Omega^{2}-i \gamma_{m} \Omega\right)$ is the mechanical response function, and $\bar{G}_{0}$ is defined as $G_{0} \bar{a}$. We choose the detuning to be $\Delta=\omega_{c}-\omega_{p} \sim \omega_{m}$.

Since the lower sideband of the laser is far detuned from the cavity resonance, we only have the upper sideband injection

$$
\hat{a}(\Omega) \approx \frac{\bar{G}_{0}}{i \gamma} \hat{x}(\Omega)+\frac{\sqrt{2 \gamma_{1}}}{\gamma} \hat{a}_{\text {in }}^{s}(\Omega) .
$$

We make use of the near-resonance approximation $\Omega-\Delta=$ $\omega \ll \gamma$. Substituting (4) into the radiation-pressure force term in (3a), the equation of motion for mechanical oscillator can be written as

$$
\chi_{\mathrm{eff}}(\omega) \hat{x}(\Omega)=-\frac{\sqrt{2 \gamma_{1}} \hbar \bar{G}_{0}}{\gamma} \hat{a}_{\mathrm{in}}^{s}(\Omega)+\hat{F}_{\mathrm{th}},
$$

in which the effective mechanical response function $\chi_{\mathrm{eff}}$ is $\chi_{\text {eff }}(\omega) \approx-2 m \omega_{m}\left(\omega-\delta+i \gamma_{m}\right)-i \hbar \bar{G}_{0}^{2} / \gamma$. In our system, the thermal force $\hat{F}_{\text {th }}$ with the spectrum density $S_{\mathrm{FF}}^{\text {th }}=$ $4 m \gamma_{m} k_{B} T \sim 10^{-30} \mathrm{~N}^{2} / \mathrm{Hz}$ [24] is negligible compared with the radiation-pressure force of the beating field with the spectrum density $S_{\mathrm{FF}}^{n} \approx 2 \gamma_{1}\left(\hbar \bar{G}_{0}\left|\delta \hat{a}_{\text {in }}^{s}\right|\right)^{2} / \gamma^{2} \gamma_{m} \sim 10^{-21} \mathrm{~N}^{2} / \mathrm{Hz}$ for measurements shown in Fig. 4.

Substituting (5) into (4) and using the relation between the transmitted field and injected field $\hat{a}_{\text {trans }}=\sqrt{2 \gamma_{2}} \hat{a}$, we have effective transmissivity

$$
t(\Omega)=2 \sqrt{\eta_{c}\left(1-\eta_{c}\right)} \frac{\Omega-\omega_{m}+i \gamma_{m}}{\Omega-\omega_{m}+i \gamma_{m}+i \Gamma_{\mathrm{opt}}},
$$

where the cavity coupling parameter is $\eta_{c}=\gamma_{1} /\left(\gamma_{1}+\gamma_{2}\right)$. The characteristic frequency $\Gamma_{\text {opt }}$ is defined to be equal to $\hbar \bar{G}_{0}^{2} / 2 m \omega_{m} \gamma$ and is tunable, principally through $\bar{G}_{0}$, which depends on the control light input power [14]. 


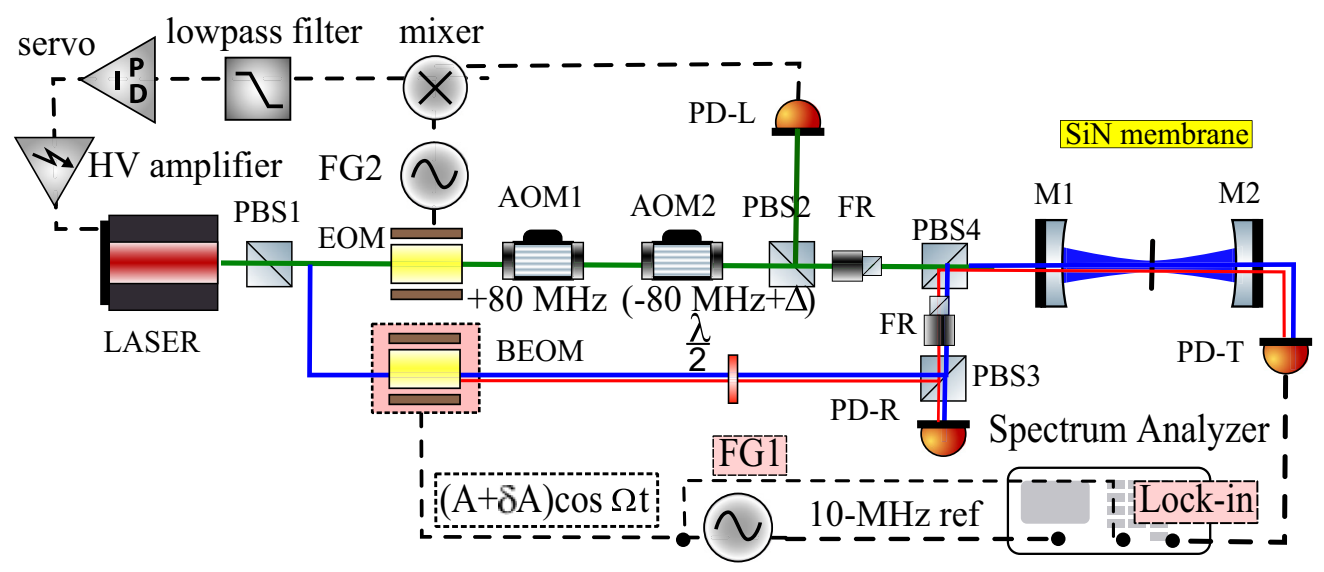

FIG. 2. (Color online) Experimental setup. The 85-mm-long cavity sits in a vacuum chamber with a central silicon nitride membrane oscillator $(1 \mathrm{~mm} \times 1 \mathrm{~mm} \times 50 \mathrm{~nm}$, effective mass $40 \mathrm{ng})$. The green line represents the locking light for stabilizing the laser frequency to the cavity resonance using the Pound-Drever-Hall method [25]. The blue line represents the control light, with polarization orthogonal to the locking light. The broadband electro-optic modulator (BEOM) generates an upper sideband from the control light, which is our signal light (red line). The $\Delta \sim 400 \mathrm{kHz}$ for the control light was created using a pair of 80-MHz acousto-optic modulators (AOMs) in the locking path. PD-L, PD-R and PD-T: photo-detectors; HV: High-Voltage; FG: function generator; PBS: polarized beam splitter; FR: faraday rotator; Lock-in: Lock-in technique for signal readout.

When we inject the signal light with squeezed added noise into the system at different frequencies, we will observe a rotation of the noise ellipse at the cavity output. The rotation angle $\theta(\Omega)$ is determined by the phase response $\phi(\Omega)$ of the cavity transmissivity $t(\Omega)$, which is given by

$$
\theta(\Omega)=\phi(\Omega)=-\arctan \frac{\Gamma_{\mathrm{opt}}\left(\Omega-\omega_{m}\right)}{\left(\Omega-\omega_{m}\right)^{2}+\gamma_{m} \Gamma_{\mathrm{opt}}},
$$

which is shown as theoretical curves in Figs. 4(b) and 4(c). When the frequency detuning $\left|\Omega-\omega_{m}\right| \gg \sqrt{\gamma_{m} \Gamma_{\text {opt }}}$, the phase $\phi(\Omega)$ of the system transmissivity can be written as

$$
\phi(\Omega)=-\arctan \left(\frac{\Gamma_{\mathrm{opt}}}{\Omega-\omega_{m}}\right),
$$

which is equivalent to the transmissivity phase response of a simple Fabry-Pérot cavity with the resonant point at $\Omega=\omega_{m}$.

Experimental scheme. In our experimental setup shown in Fig. 2, weak signal light is generated by passing the carrier control light through a broadband electro-optic modulator (BEOM). The BEOM modulates the control light and generates an upper sideband, which is our signal field. The lower sideband $\omega_{p}-\Omega$ generated by the BEOM is far detuned from the cavity resonance, so it is totally reflected and can be neglected at the transmission port. This method guarantees a common optical path for the signal light and the control light so as to avoid the fluctuating phase difference from an unlocked optical path. By adding random noise $\delta A$ to the voltage amplitude $A$, we increase the amplitude uncertainty of the signal light to simulate the phase-squeezed light where the amplitude noise is larger than the phase noise.

The core elements of our OMIT apparatus consist of an 85-mm high-finesse optical cavity with a high-stress silicon nitride membrane, which has a quality factor of $\sim 1.5 \times 10^{6}$ at the mechanical resonance. By changing the frequency separation between the signal field and control field, we observed the angle rotation of noise ellipses of the signal light, which is shown in Fig. 4(d).
Our optical cavity was mounted on an Invar spacer machined by electrical discharge machining with accuracy of $0.1 \mu \mathrm{m}$ and fixed in a vibration-isolated vacuum tank. The M1 and M2 were clamped at the ends of the spacer. We built an overcoupled cavity. The transmissivity $T 1$ of $\mathrm{M} 1$ was chosen to be much larger than that of $\mathrm{M} 2\left[T_{1}=245.1 \pm 2.8 \mathrm{ppm}\right.$, $T_{2}=16.93 \pm 0.20 \mathrm{ppm}$, where ppm denotes parts per million $\left.\left(10^{6}\right)\right]$. This experiment was conducted at room temperature using a 1064-nm Nd:YAG laser.

The mechanical oscillator in this study was a high- $Q$-factor stoichiometric silicon nitride membrane window. In order to adjust the position and alignment of the membrane in the vacuum, the membrane frame was bonded onto a piezoelectric actuator with Yacca gum, a natural resin with low intrinsic loss [26]. After gluing, we measured the quality factor of the membrane with a He-Ne laser to characterize the extra mechanical loss $\gamma_{\text {gas }}$ introduced by the background gas. When the background gas pressure $P_{\text {gas }}$ was smaller than $3 \times 10^{-5}$ mbars, the gas damping was negligibly small and the membrane quality factor was $\sim 1.5 \times 10^{6}$ at its mechanical resonance $\sim 400 \mathrm{kHz}$. (see Appendix in Ref. [27]).

Optomechanically induced transparency. In our system, the linewidth of the OMIT cavity can be changed in two ways (a) The input power of the control light can be adjusted by a halfwave plate before a polarized beam splitter (PBS3 in Fig. 2). (b) The coupled cavity linewidth $\gamma$ and the optomechanical coupling strength $G_{0}$ can be tuned by changing the position and alignment of the membrane in the cavity [22,23] (see Appendix B in [27]). We achieved a widely tunable linewidth of the OMIT cavity changing from $3 \mathrm{~Hz}$ to several hundred hertz.

In order to achieve an extremely narrow linewidth, we tuned the position and alignment of the membrane and reduced the control light input power until the characteristic frequency $\Gamma_{\text {opt }}$ was close to the mechanical linewidth $\gamma_{m}$. In Fig. 3 we show the experimental results for the lowest linewidth $3-15 \mathrm{~Hz}$. Here we define an normalized transmissivity amplitude as 

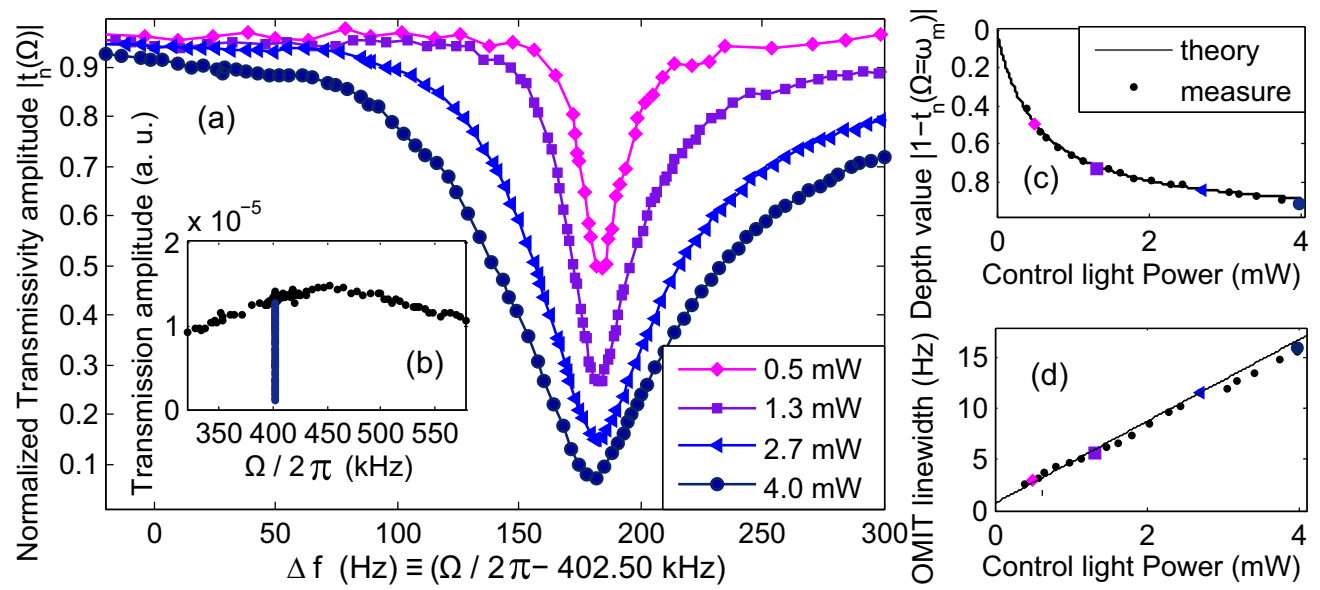

FIG. 3. (Color online) Detected OMIT transmissivity. (a) Normalized transmissivity amplitude $\left|t_{n}(\Omega)\right|$ vs frequency offset $\Delta f$ (Hz), where $\Delta f \equiv \Omega / 2 \pi-402.5 \mathrm{kHz}$. The control light powers were $0.5,1.3,2.7$, and $4.0 \mathrm{~mW}$, respectively. (b) Transmissivity amplitude vs frequency difference $\Omega / 2 \pi(\mathrm{kHz})$ in a span of $200 \mathrm{kHz}$. The coupled cavity full linewidth was $170 \mathrm{kHz}$ in this measurement. (c) Normalized transmissivity amplitude dip depth value $\left|1-t_{n}\left(\Omega=\omega_{m}\right)\right|$ vs the control light input power. (d) The OMIT cavity full linewidth vs the control light input power. The full linewidth data correspond to the Lorentzian transmissivity of the OMIT cavity. In this measurement, the mechanical resonance frequency was $\omega_{m}=2 \pi \times 402.7 \mathrm{kHz}$.

$t_{n}(\Omega) \equiv t(\Omega) / t_{0}$, where $t_{0}$ is the transmissivity amplitude of the signal light in the absence of the control field. The experimental data points in Figs. 3(c) and 3(d) are well matched with the theoretical model shown as the black solid lines.

Frequency-dependent noise ellipse rotation. The above results show that OMIT effect can be used to create cavities with tunable linewidth down to a few hertz. We now demonstrate that such cavities have the appropriate phase response and that they rotate the angle of the noise ellipse of the signal light as required for one simple filter cavity.

In order to characterize the noise ellipse rotation of the signal light in a phasor diagram, we tune the OMIT cavity linewidth to several hundred hertz and demonstrate the noise ellipse rotation in the phasor diagram. The phase response and the rotation angles of the noise ellipses are detected by lock-in technique (see Fig. 2 and [21]). We take results of the coupled cavity with a linewidth of $\sim 600 \mathrm{~Hz}$ as an example.

Figures 4(a) and 4(b) show the experimental results of the amplitude $|t(\Omega)|$ and the phase $\phi(\Omega)$ of the OMIT cavity transmission, respectively. The phase drop in the vicinity of the OMIT cavity $\left(\left|\Omega-\omega_{m}\right|<\sqrt{\gamma_{m} \Gamma_{\text {opt }}}\right)$ resonance was measured and shown in Appendix $\mathrm{C}$ of [27].

Figures 4(c) and 4(d) are the measured rotation angles $\theta(\Omega)$ and the corresponding normalized noise ellipses in the phasor diagram [28], respectively. As shown in Fig. 4(c), the measured results for angle rotation of the noise ellipses match well both

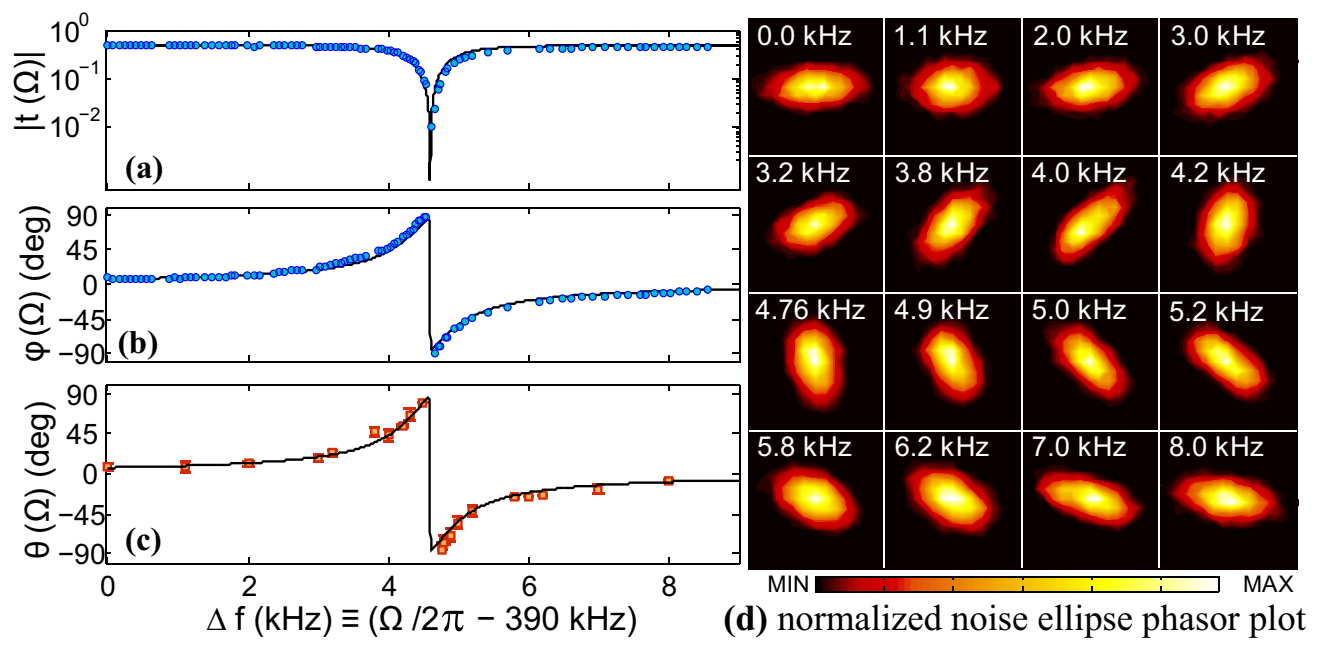

FIG. 4. (Color online) (a) The OMIT cavity transmissivity amplitude $|t(\Omega)|$, (b) phase $\phi(\Omega)$, and (c) rotation angle $\theta(\Omega)$ of the noise ellipse as a function of $\Delta f(\mathrm{kHz})$. The frequency offset is defined as $\Delta f \equiv \Omega / 2 \pi-390 \mathrm{kHz}$. In this measurement, the mechanical resonance frequency was $\omega_{m}=2 \pi \times 394.6 \mathrm{kHz}$. (d) Contour plots of the normalized noise ellipse in the phasor diagram at different frequency offsets $\Delta f$ corresponding to (c). In each phasor diagram, the horizontal axis is the amplitude quadrature and the vertical axis is the phase quadrature. The frequency offset of the OMIT cavity resonance is $4.6 \mathrm{kHz}$. 
the theoretical model and the previous measurement of the phase $\phi(\Omega)$.

Conclusion. We have shown an extremely narrow cavity linewidth created by optomechanical interaction in an optical cavity with a silicon nitride membrane in the middle. Classical light with a noise ellipse simulating quantum squeezed light was injected into the cavity. It demonstrates the frequency-dependent noise ellipse rotation. The rotation angle follows the theoretical prediction in the detection band of advanced gravitational-wave detectors. To use the current setup to develop a system for realizing a frequency-dependent squeezed vacuum in GW detectors in the future, it will be necessary to cool the resonator to the $\mathrm{mK}$ temperature range and dilute the mechanical losses by optical springs, as discussed in Refs. [7,18].

We thank Haixing Miao, Yanbei Chen, Lisa Barsotti, Stefan Danilishin, and David McClelland for useful discussions. We thank the technicians Gary Light and Mark Dickinson for technical support. We would like to thank the LIGO Scientific Collaboration, Gingin International Advisory, and our collaborators, especially Stefan Gosler, Gregg Harry, Bill Kells, Pierre-Francois Cohadon, and Antoine Heidmann for useful advice. This research was supported by the Australian Research Council (Grants No. DP120104676 and No. DP120100898).
[1] V. B. Braginsky, Sov. Phys. JETP 26, 831 (1968); Physical Experiments with Test Bodies, NASA Technical Translation TT F-672 (U.S. Technical Information Service, Springfield, VA, 1972).

[2] V. B. Braginsky and Yu. I. Vorontsov, Sov. Phys. Usp. 17, 644 (1975).

[3] H. J. Kimble, Y. Levin, A. B. Matsko, K. S. Thorne, and S. P. Vyatchanin, Phys. Rev. D 65, 022002 (2001).

[4] S. Chelkowski et al., Phys. Rev. A 71, 013806 (2005).

[5] M. S. Stefszky et al., Class. Quantum Gravity 29, 145015 (2012).

[6] M. Evans, L. Barsotti, P. Kwee, J. Harms, and H. Miao, Phys. Rev. D 88, 022002 (2013).

[7] Y. Ma, S. L. Danilishin, C. Zhao, H. Miao, W. Z. Korth, Y. Chen, R. Ward, and D. G. Blair, arXiv:1402.4897.

[8] K.-J. Boller, A. Imamoglu, and S. E. Harris, Phys. Rev. Lett. 66, 2593 (1991).

[9] M. D. Lukin, Rev. Mod. Phys. 75, 457 (2003).

[10] M. D. Lukin and A. Imamoglu, Nature (London) 413, 273 (2001).

[11] M. Fleischhauer, A. Imamoglu, and J. P. Marangos, Rev. Mod. Phys. 77, 633 (2005).

[12] A. Kasapi, M. Jain, G. Y. Yin, and S. E. Harris, Phys. Rev. Lett. 74, 2447 (1995).

[13] E. E. Mikhailov, K. Goda, T. Corbitt, and N. Mavalvala, Phys. Rev. A 73, 053810 (2006).
[14] S. Weis et al., Science 330, 1520 (2010).

[15] M. Karuza et al., Phys. Rev. A 88, 013804 (2013).

[16] D. E. Chang, K.-K. Ni, O. Painter, and H. J. Kimble, New J. Phys. 14, 045002 (2012).

[17] K.-K. Ni, R. Norte, D. J. Wilson, J. D. Hood, D. E. Chang, O. Painter, and H. J. Kimble, Phys. Rev. Lett. 108, 214302 (2012).

[18] W. Z. Korth et al., Phys. Rev. A 88, 033805 (2013).

[19] B. M. Zwickl et al., Appl. Phys. Lett. 92, 103125 (2008).

[20] A. M. Jayich et al., New J. Phys. 14, 115018 (2012).

[21] A. H. Safavi-Naeini et al., Nature (London) 472, 69 (2011).

[22] C. Biancofiore et al., Phys. Rev. A 84, 033814 (2011).

[23] A. M. Jayich, J. C. Sankey, B. M. Zwickl, C. Yang, J. D. Thompson, S. M. Girvin, A. A. Clerk, F. Marquardt, and J. G. E. Harris, New J. Phys. 10, 095008 (2008).

[24] C. Kittel and H. Kroemer, Thermal Physics (Freeman, San Francisco, 1980).

[25] E. D. Black, Am. J. Phys. 69, 79 (2001).

[26] S. W. Schediwy, S. Gras, L. Ju, and D. G. Blair, Rev. Sci. Instrum. 76, 026117 (2005).

[27] See Supplemental Material at http://link.aps.org/supplemental/ 10.1103/PhysRevA.89.041802 for discussions and accessorial measurements of the parameters of the coupled cavity system.

[28] C. W. Gardiner and P. Zoller, Quantum Noise (Springer, Berlin, 2004). 